\title{
Dor torácica aguda: enfermeiro desafiando uma patologia tempo dependente nas portas de entrada hospitalares
}

Acute chest pain: nurses challenging a time-dependent pathology at the hospital entrance doors Dolor torácico agudo: enfermero desafiando una patología dependiente del tiempo en las puertas de entrada hospitalarias

\section{Angélica Zanettini I, Marisa Basegio Carretta Diniz ${ }^{\text {II }}$, Gabriela Stochero ${ }^{\text {III }}$, Juliane Christofari da Silva ${ }^{\mathrm{IV}}$, Camila Marcon ${ }^{\mathrm{V}}$, Viviane Pecini da Cunhavi}

Resumo: Objetivo: avaliar a percepção dos enfermeiros diante do paciente com dor torácica nas portas de entrada do Serviço de Urgência e Emergência de um hospital geral. Método: estudo exploratório, descritivo, com abordagem qualitativa, realizado com 10 enfermeiros em um hospital geral de grande porte do Rio Grande do Sul, em 2018. A coleta de dados ocorreu através de entrevistas semiestruturadas, estudadas conforme análise de conteúdo. Resultados: constataram-se dificuldades no entendimento da Classificação de Risco, questões estruturais e organizacionais e a falta de profissionais qualificados. Como potencialidades, atendimento de maneira ágil, buscando a mais correta identificação dos sinais apresentados pelo paciente e a realização do eletrocardiograma no tempo adequado. Conclusão: evidenciou-se que o enfermeiro percebe estar apto para tomar decisões rápidas e precisas por meio do conhecimento de protocolos assistenciais. Porém, verifica-se a interferência de processos como o subdimensionamento de pessoal e a demanda excessiva dos serviços de porta de entrada.

Descritores: Cardiopatias; Enfermagem; Emergências; Dor no Peito

\footnotetext{
I Enfermeira. Especialista em Cardiologia pela Universidade de Passo Fundo (UPF). Chapecó. Santa Catarina. Brasil. E-mail: angeliica.zanettini@gmail.com. ORCID: https://orcid.org/0000-0003-1712-9073.

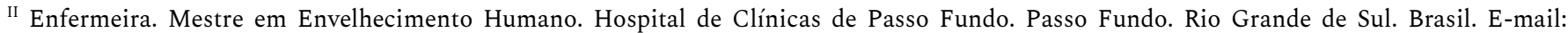
marisa.diniz@hcpf.com.br. ORCID https: //orcid.org/0000-0002-3542-313X.

${ }^{\text {III }}$ Enfermeira. Especialista em Cardiologia pela Universidade de Passo Fundo (UPF). Hospital de Clínicas de Passo Fundo. Passo Fundo. Rio Grande de Sul. Brasil. E-mail: juliane_c_s@yahoo.com.br. ORCID: https://orcid.org/0000-0003-1503-4150.

${ }^{\text {IV }}$ Enfermeira. Especialista em Cardiologia pela Universidade de Passo Fundo (UPF). Hospital Unimed Noroeste. Ijuí. Rio Grande de Sul. Brasil. E-mail: gabi_stochero@hotmail.com. ORCID: https: //orcid.org/0000-0003-0660-3622.

${ }^{\vee}$ Enfermeira. Especialista em Oncologia pela Universidade de Passo Fundo (UPF). Hospital Unimed Chapecó. Chapecó. Santa Catarina. Brasil. E-mail: milamarcon_md@hotmail.com. ORCID: https: //orcid.org/0000-0003-4338-2857.

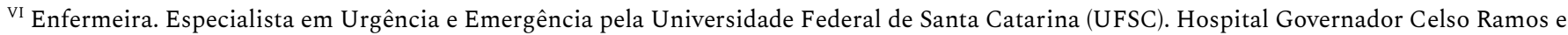
CEPON em Florianópolis. Santa Catarina. Brasil. E mail: vipecini@gmail.com. ORCID: https: //orcid.org/0000-0003-2026-5981.
} 


\begin{abstract}
Objective: to assess the perception of nurses in the face of patients with chest pain at the entrance doors of the Urgent and Emergency Service in a general hospital. Method: exploratory and descriptive study, with a qualitative approach, carried out with 10 nurses in a large general hospital in Rio Grande do Sul, in 2018. Data collection occurred through semi-structured interviews, studied according to content analysis. Results: there were difficulties in understanding the Risk Classification, structural and organizational issues, besides the lack of qualified professionals. As for potentialities, care in a quick way, seeking the most correct identification of the signs presented by the patient and the accomplishment of the electrocardiogram in the appropriate time. Conclusion: it was evidenced that the nurse perceives to be able to make quick and accurate decisions through the knowledge of care protocols. Nevertheless, there is interference from processes such as undersizing of personnel and the excessive demand for entrance door services.
\end{abstract}

Descriptors: Heart Diseases; Nursing; Emergencies; Chest Pain

Resumen: Objetivo: evaluar la percepción de los enfermeros ante los pacientes con dolor torácico en las puertas de entrada del Servicio de Urgencia y Emergencia de un hospital general. Método: estudio exploratorio, descriptivo, con enfoque cualitativo, realizado con 10 enfermeros en un gran hospital general en Rio Grande do Sul, en 2018. La recolección de datos ocurrió mediante entrevistas semiestructuradas, estudiadas según el análisis de contenido. Resultados: se encontraron dificultades para comprender la Clasificación de Riesgos, las cuestiones estructurales y organizacionales y la falta de profesionales calificados. Como potenciales, atención desarrollada rápidamente, buscando la identificación más correcta de los signos presentados por el paciente y la realización del electrocardiograma a tiempo. Conclusión: se evidenció que el enfermero percibe la capacidad de tomar decisiones rápidas y precisas mediante el conocimiento de protocolos asistenciales. Sin embargo, hay interferencia de procesos como el subdimensionamiento de personal y la excesiva demanda de servicios de admisión.

Descriptores: Cardiopatías; Enfermería; Emergencias; Dolor en el Pecho

\title{
Introdução
}

A dor torácica na emergência representa um grande desafio e seu diagnóstico pode ser favorecido pelas particularidades de sua localização e da sintomatologia associada. Geralmente, refere-se à dor ou desconforto na região do tórax de origem cardíaca ou não cardíaca. Esta pesquisa tem foco na dor cardíaca, que pode ser identificada com características típicas. Sua investigação deve ser criteriosa, visto que algumas etiologias são potencialmente fatais se não prontamente reconhecidas. ${ }^{1}$

Quando a dor é de foco cardíaco, a maioria dos pacientes na avaliação da gravidade relatou fortes dores, como sensação de aperto, queimação, pontadas ou opressão com foco na região pré-cordial. Esse desconforto pode ou não ter irradiação. Quando irradiado segue para 
3 | Zanettini A, Diniz MBC, Stochero G, Silva JC, Marcon C, Cunha VP

lugares como mandíbula e membros superiores, acompanhado de sudorese, náuseas e síncope. Sendo assim, todo e qualquer tipo de dor torácica necessita de atendimento rápido para investigação e conduta adequada. ${ }^{2}$

O infarto agudo do miocárdio (IAM) é uma doença isquêmica do coração, causadora de dor e que ocorre secundariamente à interrupção aguda do fornecimento de sangue e oxigênio por meio das coronárias. A destruição do músculo do coração é determinada, geralmente, por depósitos de placas de ateroma, embolo ou trombo, causando uma lesão permanente no músculo do miocárdio. A dimensão dessa necrose dependerá do tamanho da artéria lesada, do tempo de desenvolvimento da obstrução e se houve circulação colateral..$^{3-4}$

O panorama epidemiológico mundial tem revelado um grande crescimento das doenças cardiovasculares $(\mathrm{DCV})$ e, por sua vez, o IAM é a patologia que aparece com mais frequência, merecendo notoriedade devido a sua alta taxa de mortalidade. Estimativas demonstram que, para o ano de 2020, o infarto seja a causa de aproximadamente 25 milhões de óbitos, com 19 milhões deles nos países de baixa e média renda, atrelada ao Sistema Único de Saúde (SUS). Isso atribuído às dificuldades de tratamento, seja nos métodos de reperfusão ou em medidas terapêuticas estabelecidas ou pela carência de Unidade de Terapia Intensiva..$^{5-6}$

Nesse contexto, a dor torácica é uma emergência cardiológica que leva o paciente a buscar os serviços de saúde. Na presença desta, deve-se realizar uma avaliação adequada para concluir o possível diagnóstico de IAM. Portanto, é de suma importância que o atendimento pré-hospitalar ocorra de maneira rápida e eficiente, resultando, assim, na redução do tempo entre o início do evento isquêmico e necrose até o tratamento. ${ }^{7}$

O enfermeiro é responsável por realizar a avaliação do risco no serviço de emergência, cabendo a ele avaliar o paciente, determinar as necessidades de prioridade no atendimento e encaminhá-lo para a área de tratamento de acordo com as suas demandas. Sendo assim, o enfermeiro, muitas vezes, é o profissional da equipe de emergência a ter o primeiro contato com 
o paciente, tendo o papel de identificar a gravidade e encaminhá-lo para os procedimentos que serão prestados de maneira ágil e eficaz.

Dessa forma, entende-se que um atendimento rápido e efetivo oferta maior sobrevida para os pacientes, além de possibilitar traçar condutas e estratégias para a resolução das alterações de maneira adequada. No contexto citado, questiona-se: qual o papel e a importância da enfermagem no cumprimento dos tempos pactuados no protocolo de dor torácica?

A Política Nacional de Humanização (PNH) propõe ações integradas que buscam modificar a assistência dos usuários nos hospitais públicos no Brasil, aprimorando a qualidade e eficiência nos serviços das instituições. ${ }^{8}$ Isso é possível por meio da implementação da classificação de risco (CR), que é um sistema dinâmico de identificação dos pacientes, atentando para as necessidades e tratamento instantâneo, decorrentes da sua situação de saúde ou sofrimento. ${ }^{9}$

A importância da realização deste estudo ratifica-se pelo fato de que na instituição em questão existe um protocolo de atendimento para a dor torácica e o instrumento utilizado como CR apresenta-se insuficiente de informações. Essa fragilidade vem justificar o interesse desta pesquisa que visa observar o conhecimento e o embasamento da prática profissional dos enfermeiros. Para isso, tem-se como objetivo avaliar a percepção dos enfermeiros diante do paciente com dor torácica nas portas de entrada do Serviço de Urgência e Emergência de um hospital geral.

\section{Método}

O presente estudo caracteriza-se por ser uma pesquisa descritiva, exploratória, de abordagem qualitativa, realizada em um hospital de grande porte do norte do Estado do Rio Grande do Sul (RS). As pesquisas qualitativas visam compreender as significações, os motivos, as aspirações, as crenças, os valores e atitudes subjetivas que podem ser observados nas falas dos 
5 | Zanettini A, Diniz MBC, Stochero G, Silva JC, Marcon C, Cunha VP

indivíduos. Já as descritivas procuram descrever, com a maior precisão possível, a ocorrência de um fato, a relação com outros, características e sua natureza. ${ }^{10}$

A pesquisa atendeu aos passos recomendados pelos Critérios Consolidados para Relatar uma Pesquisa Qualitativa (COREQ). ${ }^{11}$ Os dados foram coletados no período de março a agosto de 2018, nos três turnos, com todos os 10 enfermeiros, totalizando 10 entrevistas.

Realizou-se a coleta dos dados, utilizando um roteiro com questões semiestruturadas, composto por perguntas abertas. A entrevista foi individual, aconteceu de forma pré-agendada, a fim de ter um momento disponível e propício. O roteiro envolvia dados sobre a principal atividade do enfermeiro na porta de entrada.

Participaram do estudo os enfermeiros que atuam nas portas de entrada da instituição de acordo com os seguintes critérios de elegibilidade: ser enfermeiro efetivo da instituição; sem restrição de idade máxima; e estar em exercício profissional no período de coleta de dados. O critério de exclusão foi: enfermeiros atuantes de outras unidades ou enfermeiros substitutos.

A coleta de dados ocorreu por meio de uma entrevista com cada participante. No primeiro momento, os entrevistados eram caracterizados em relação à categoria profissional, titulação e tempo de serviço. $\mathrm{Na}$ segunda parte da entrevista, foram exploradas questões referentes à temática, como o conhecimento sobre identificação da dor torácica, o tempo de realização de eletrocardiograma (ECG), as dificuldades encontradas nas portas de entrada para aplicação da CR e a avaliação dos protocolos assistências.

Todas as falas foram gravadas em smartphone, transcritas na íntegra e estudadas a partir da análise de conteúdo. ${ }^{12}$ A duração média de cada uma foi de 30 minutos. A fim de assegurar o anonimato dos entrevistados, os recortes de suas falas foram identificados pela codificação do nome de 10 partes anatômicas do coração, sendo elas Artéria Coronária Direita (ACD), Artéria Circunflexa (ACX), Artéria Descendente Anterior (ADA), Aorta, Veia Cava Superior (VCS), Veia Cava Inferior (VCI), Valva Tricúspide (V. Tricúspide), Valva Aórtica (V. 
Dor torácica aguda: enfermeiro desafiando uma patologia tempo dependente nas... 6

Aórtica), Valva Pulmonar (V. Pulmonar), Valva Mitral (V. Mitral). Justifica-se a escolha dos nomes pelo fato de estarem diretamente correlacionados com a patologia em foco.

Os dados foram organizados e submetidos aos procedimentos analíticos; em seguida, realizou-se a análise a partir das transcrições das entrevistas, utilizando-se o método de análise de conteúdo. ${ }^{12}$ Esta é um conjunto de técnicas de análise das comunicações com o intuito de obter, por meio de procedimentos sistemáticos e objetivos de descrição, a inferência de conhecimentos relativos às condições de produção/recepção dessas mensagens.

Inicialmente, realizaram-se a pré-análise com leitura flutuante dos dados extraídos nas entrevistas, a construção de tabela com os dados coletados e a escolha dos documentos para a constituição dos dados para os procedimentos analíticos; em seguida, explorou-se o material de análise, organizando os dados em três categorias que serão apresentadas e discutidas por meio da revisão de literatura.

Vale destacar que a pesquisa em questão foi realizada após a aprovação do Comitê de Ética em Pesquisa da Universidade de Passo Fundo - UPF, sob o parecer nº 2.572 .484 e o protocolo CAAE 83480218.1.0000.5342, no dia 31 março de 2018, e da instituição hospitalar. Todos os participantes assinaram o Termo de Consentimento Livre e Esclarecido após serem informados quanto ao objetivo da pesquisa, o meio de divulgação dos resultados e os direitos, como confidencialidade dos dados obtidos, anonimato e voluntariedade em participar do estudo. Os princípios éticos e legais, o sigilo e o anonimato, conforme a Resolução nº 466/2012, do Conselho Nacional de Saúde, ${ }^{13}$ foram respeitados.

\section{Resultados e discussão}

A partir da análise das informações, pode-se avaliar a percepção dos enfermeiros diante do paciente com dor torácica por meio de três categorias: compreendendo a dor torácica na sua subjetividade; enfermeiro avaliando a dor torácica baseado em protocolos assistenciais; o 
enfermeiro desafiando a realidade atual do serviço de urgência e emergência perante as adversidades assistenciais.

Participaram do presente estudo 10 enfermeiros, seis do sexo feminino e quatro do sexo masculino, todos efetivos nos cargos. As idades variaram entre 22 e 56 anos, média de 31,6 \pm 10,26 anos. O tempo de atuação na profissão variou entre quatro meses e 38 anos, com média de $8,5 \pm 11,31$ anos e tempo de atuação na emergência entre quatro meses e 10 anos, sendo a média de 2,3 \pm 3,03 anos. Quanto à formação, sete enfermeiros possuem especialização, dois estão cursando pós-graduação em paciente crítico e um não possui especialização.

\section{Compreendendo a dor torácica na sua subjetividade}

Os fatores de riscos podem ser divididos em duas classes, os modificáveis e os não modificáveis. Os modificáveis estão relacionados com hábitos de vida, sendo eles hipertensão arterial sistêmica, tabagismo, glicemia elevada, dislipidemia, uso de álcool, estresse, dislipidemia, inatividade física e obesidade. Já os não modificáveis incluem o sexo, idade e a hereditariedade. Esses são responsáveis por mais de $40 \%$ da mortalidade global. ${ }^{14}$

As DCV são crônicas e se caracterizam por longo período de latência, fazendo com que os pacientes só descubram sua gravidade quando os sintomas já estão exacerbados e a doença em período avançado. Nesse momento, podem já existir lesões irreversíveis, gerando, assim, amplas complicações. Em razão desses fatores, essas doenças ganham espaço no perfil epidemiológico de morbimortalidade por cardiopatia. ${ }^{15}$

O principal sintoma que determina a investigação diagnóstica diante de um quadro de possível SCA é a dor torácica. Os fatores desencadeantes são o esforço físico (mais comum) ou emoção. O fato de a dor não ser desencadeada por algum desses dois fatores não exclui a possibilidade que seja de origem cardíaca. Diante da fala da ACX, consegue-se observar que a dor torácica se torna a principal queixa relatada: 
Dor torácica aguda: enfermeiro desafiando uma patologia tempo dependente nas... 8

Queixa de dor torácica, ou queixa de dor que vai do mento ao umbigo, início súbito ou tardio, enfim, mas mais o súbito, intensidade forte. $\mathrm{Na}$ verificação dos sinais, saturação baixa, frequência cardíaca alterada alta, geralmente é assim. (ACX)

Fica evidente que a dor intensa que vem descrita pelo paciente é determinante para a avaliação, como também na CR. O papel do enfermeiro é fundamental nesse processo, uma vez que a compreensão e o entendimento da possível origem da dor colocam o paciente em um critério de risco diferenciado e tempo dependente. Aqui, a subjetividade da dor necessita ser bem conduzida, visto que impõe ao profissional a necessidade de avaliar e classificar o paciente com a objetividade que o caso merece.

Segundo a Sociedade Brasileira de Cardiologia (SBC), a SCA tem uma descrição clássica, que se apresenta com dor, desconforto, queimação, sensação opressiva localizada na região precordial ou retroesternal, podendo ter irradiação para o ombro e/ou braço esquerdo, braço direito, pescoço ou mandíbula, acompanhada frequentemente de diaforese, náuseas, vômitos e/ou dispneia. ${ }^{16} \mathrm{~A}$ duração dos sintomas pode persistir por alguns minutos ou até mais que 30 minutos, se for IAM. No entanto, algumas vezes, poderá apresentar uma queixa atípica, por exemplo, mal-estar, indigestão, fraqueza ou apenas sudorese, sem dor. ${ }^{1,16}$

Nesse contexto, é condição determinante que o profissional que trabalha nas portas de entrada dos serviços hospitalares com a CR tenha conhecimento acerca dos fatores de risco e comorbidades. Dentre eles, idade avançada, sexo, diabetes mellitus, dor torácica característica, pois estes impactam na evolução da doença e no desfecho. Portanto, é relevante ter o conhecimento dos fatores para realizar a CR de maneira fidedigna:

Quando o paciente chega relatando uma dor como se fosse uma dor aguda na região do lado esquerdo, irradiando para o braço, ou então se tem alguma pré-disposição, se for mulher, diabético ou idade avançada. (ADA)

Nesses casos, a identificação da gravidade na classificação deve ser elencada de maneira rápida e logo acionado o serviço especializado. Quando um IAM é identificado, o tempo é 
9 | Zanettini A, Diniz MBC, Stochero G, Silva JC, Marcon C, Cunha VP

determinante para o sucesso do atendimento e efetividade do tratamento, percorrendo desde o seu diagnóstico até a minimização dos riscos de vitalidade. Isso pode aumentar o prognóstico para os pacientes que apresentam tais sintomas.

A avaliação criteriosa dos enfermeiros nas portas de entrada aos pacientes com dor torácica é primordial, principalmente na contribuição de um diagnóstico rápido e eficaz. Deve ser organizada de forma que priorize o paciente em questão. O fluxo desse processo precisa acontecer de forma dinâmica entre o acolhimento e CR. A classificação inclui a entrevista, a identificação de sinais/sintomas, realização do ECG e a coleta de marcadores de necrose miocárdica. Busca-se, com isso, sempre aperfeiçoar o tempo porta-agulha de 30 minutos para potencializar a resolutividade, garantindo, desse modo, uma assistência de qualidade. ${ }^{17}$

Neste estudo, há relatos de enfermeiros que evidenciam a importância da realização da triagem de maneira ágil para identificação do IAM:

Há vários sinais, na emergência a gente identifica através da triagem, dos sinais vitais, quando o paciente chega com uma dor aguda, uma dor em queimação, se desloca para o ombro ou pescoço, quando está sudorético, ou pela própria entrevista a gente já consegue identificar e passar para atendimento. (V. Tricúspide)

[...] tudo depende da triagem, da agilidade, do feeling de você pegar a sintomatologia, às vezes ele não te fala que está com a dor precordial, relata uma epigastralgia, tu começa associar, está hipertenso, eu acho que a gente tem um bom resultado no final dos segmentos dos casos [...] (V. Mitral)

Contextualizando com o exposto, o profissional da saúde tem a possibilidade de utilizar os referenciais disponibilizados pela instituição como o protocolo de dor torácica e as diretrizes da SBC para facilitar a priorização no atendimento. Por isso, a CR é uma importante ferramenta nesse processo, sendo aplicada nos serviços de urgência e emergência. Quando a necessidade clínica excede a oferta, utiliza-se a classificação para assegurar a atenção médica com um tempo adequado, organização do fluxo para os pacientes e identificação precoce de casos agudos. 
Dor torácica aguda: enfermeiro desafiando uma patologia tempo dependente nas... 10

\section{Enfermeiro avaliando a dor torácica baseado em protocolos assistenciais}

Atualmente, no Brasil, a excessiva procura pelo atendimento de emergência é um problema que acarreta em acúmulo de tarefas. Uma necessidade maior de consultas contribui para o aumento dos custos de atendimento, gera sobrecarga para os profissionais da equipe de saúde e algumas vezes acaba ultrapassando a capacidade na qual os serviços estão organizados. ${ }^{1}$

No intuito de garantir a qualidade de atendimento prestado aos usuários, de forma objetiva e rápida, o enfermeiro realiza a CR. No hospital em foco, utiliza-se o sistema de triagem de Manchester (STM). Neste, o classificador avalia o paciente de acordo com a queixa clínica, buscando identificar os sinais e sintomas, pois o número de pessoas que procuram as portas de entrada com queixas compatíveis, com a possibilidade de serem decorrentes de uma SCA ou de outras doenças cardiovasculares graves, é cada vez maior. ${ }^{18}$

A CR necessita priorizar o atendimento à dor torácica. O método do STM preconiza que o tempo máximo de espera do paciente, entre a chegada à unidade de emergência e a classificação, não ultrapasse 10 minutos, visto que se sabe que a maioria dos casos de morte por IAM ocorre nas primeiras horas do início dos sintomas. A primeira hora do evento é responsável por 40 a $65 \%$ dos óbitos, o que converge para um percentual de $80 \%$ nas primeiras 24 horas. ${ }^{1,18}$

Os profissionais afirmaram que a CR acontece de maneira ágil, buscando a mais correta identificação dos sinais apresentados pelo paciente. Prioriza-se, assim, o atendimento emergencial para a cardiologia:

No meu setor eu digo até $10 \mathrm{~min}$, porque o paciente que chega referindo dor torácica, passo imediatamente para a classificação e encaminho para o eletro. (ACD)

[...] no nosso serviço em até 10 minutos ele tem que ser atendido, ele é classificado em vermelho ou laranja, mas geralmente é vermelho, porque ele faz a triagem, o Manchester classifica em vermelho [...] (ACX) 
11 | Zanettini A, Diniz MBC, Stochero G, Silva JC, Marcon C, Cunha VP

Além da triagem, é importante demonstrar aos enfermeiros as reais necessidades da aplicação e execução do protocolo simultaneamente com suas competências na prática clínica. Isso porque esses profissionais, além de auxiliarem no diagnóstico, direcionam a conduta terapêutica, diminuindo o tempo entre a disponibilização de medicamentos e solicitação de exames.

No que se refere aos eventos coronarianos, a redução de casos como o IAM acontece devido ao uso de diretrizes com evidências científicas comprovadas. Além disso, o uso dos protocolos se torna essencial para aperfeiçoar a qualidade e a efetividade do atendimento.

A realização de procedimentos essenciais, como o ECG e a coleta de marcadores cardíacos, aperfeiçoa o potencial de resolutividade e garante uma assistência de qualidade, priorizando a diminuição do tempo porta-balão. ${ }^{1-2}$

Corroborando com essa necessidade, a SBC considera ser primordial que o diagnóstico de IAM, por meio do ECG com 12 derivações, seja realizado em um prazo não superior a 10 minutos da chegada do paciente. Esse é considerado o tempo-ouro para o início da terapêutica adequada. ${ }^{16}$ Em pacientes com sintomas sugestivos, a elevação do segmento ST tem especificidade de $91 \%$ e sensibilidade de $46 \%$ para diagnóstico de IAM. ${ }^{16,18}$

Ao serem indagados sobre o tempo para a realização do ECG, quando classificado o paciente com dor torácica, obtiveram-se as seguintes respostas:

O ideal é que seja menos que 10 minutos, eu acho que a partir do momento que o enfermeiro viu, eu posso dizer que é 10 minutos, acho que a maior perda de tempo é até o enfermeiro vê esse paciente. (ACD)

Se a dor do paciente é muito intensa, muito sudorético e assim que a gente vê pelo perfil dele é imediato. Às vezes cinco minutos para fazer, de cinco a dez minutos. (Aorta)

A partir deste estudo, foi possível evidenciar e observar que ECG está de acordo com o preconizado pela SBC, principalmente pelo fato de que, no serviço estudado, esse exame faz parte de um protocolo. Dessa forma, possibilita-se a autonomia da solicitação pelo enfermeiro. 
Dor torácica aguda: enfermeiro desafiando uma patologia tempo dependente nas... 12

Nesse contexto, emergem como tecnologia do cuidado e instrumento para o embasamento da prática de enfermagem os protocolos assistenciais. Estes visam suprir as necessidades dos enfermeiros, auxiliando para a tomada de decisão durante a CR e orientando o percurso que deve ser percorrido com o paciente perante as boas práticas baseadas em evidências.

Outro indicador importante são os marcadores séricos de necrose miocárdica ou biomarcadores, pois com o ECG conseguem complementar a avaliação inicial realizada no paciente que não apresentou elevação do segmento ST. Nos pacientes com o segmento ST, sua relevância se torna menor, uma vez que não são essenciais para o diagnóstico.

Tradicionalmente, são mensuradas as enzimas CK-MB e troponina $\mathrm{I}$ e $\mathrm{T}$, sendo a troponina considerada o marcador padrão-ouro para o diagnóstico de IAM. Logo, deve ser realizado o exame na chegada, em 2 horas e 4 horas após, necessitando permanecer sob monitorização contínua. No local de realização da presente pesquisa, realizam-se os exames de CK-MB e troponina $\mathrm{T}^{19}$

O tratamento precoce é medida salvadora de vidas e deve ser iniciado precocemente. Quando indicada a terapia de reperfusão em pacientes com supra de segmento ST, a meta para a trombólise coronariana é de 30 minutos e o tempo porta-balão não deve ser superior a 90 minutos. Nos casos de infarto de grande extensão ou início dos sintomas há menos de duas horas, o recomendado é um máximo de 60 minutos..$^{18,20}$

Em relação ao tempo de resultado dos biomarcadores, percebe-se um desconhecimento dos enfermeiros referente ao tempo preconizado. Este está no protocolo de dor torácica implementado pela instituição, que se refere a 45 minutos, como se constatou nas falas a seguir:

Geralmente quando e suspeita de infarto, a gente já liga para o laboratório, eles já descem, já coletam, mais uma hora/duas horas por aí. (ADA)

Não faço ideia. Teríamos que ter um indicador para sabermos bem certinho os tempos. (ACX) 
O atendimento rápido e adequado dos pacientes que apresentam SCA é uma das maiores preocupações dos responsáveis pelos serviços de emergência, pois interfere tanto na diminuição de riscos e agravos como em gastos para instituição hospitalar, na medida em que se evitam terapêuticas e internações inadequadas. A falta de domínio e de fundamentação dos enfermeiros das portas de entrada, quanto à abordagem do paciente, ocasionará um atraso do encaminhamento adequado, gerando, assim, um aumento proporcional do risco de mortalidade. ${ }^{1}$

O enfermeiro desafiando a realidade do serviço de urgência e emergência perante as adversidades assistenciais

O início do atendimento ao paciente com SCA começa nas estratégias de abordagem e recebimento. Esse serviço é realizado por meio de uma equipe treinada na recepção e CR que priorize e reconheça os casos de dor torácica.

Assim sendo, constata-se que o enfermeiro assume um importante papel nas portas de entrada, no que se refere à coordenação da equipe e à gerência do cuidado aos pacientes com necessidades complexas. Isso demanda conhecimento científico, capacidade de manejo tecnológico, análise crítica e agilidade.

Para a eficiência da aplicabilidade dos fluxogramas e CR, é importante que o enfermeiro tenha domínio de todas as partes componentes dos protocolos assistenciais. Soma-se a isso a necessidade de desempenhar escuta qualificada e atendimento humanístico, resultando em um atendimento completo. A vulnerabilidade e o risco do indivíduo são considerados nesse contexto. Portanto, o diagnóstico é feito pelos profissionais que levam em consideração a história de quem está sendo cuidado, de modo a possibilitar decisões compartilhadas. ${ }^{21}$

Os serviços de urgência e emergência vêm historicamente funcionando como porta de entrada do SUS. Devido à grande procura por atendimentos, essa porta de entrada acaba recebendo desde pacientes graves até as ocorrências mais simples, o que resulta em dificuldades 
Dor torácica aguda: enfermeiro desafiando uma patologia tempo dependente nas... 14

no dimensionamento de pessoal. Observou-se essa demanda excessiva no relato dos enfermeiros a seguir:

A principal dificuldade é o acúmulo de pessoas para triagem, aí até chegar essa informação lá na triagem às vezes se perde um bom tempo, pelo fato de estar acumulando muitas consultas, não emergências [...]. (V. Mitral)

Muita demanda clínica de pacientes que não necessitariam estar na emergência, se reduzissem os 60-80 atendimentos para os pacientes que seriam emergência e urgência poderia ser feito o atendimento integral, completo e rápido, diferente de atender uma demanda absurda que se tem hoje. (V. Pulmonar)

As mudanças no cenário epidemiológico e demográfico devido à persistência de doenças infecciosas e parasitárias, a prevalência das Doenças Crônicas Não Transmissíveis (DCNT) associadas às situações de violência e doenças em processos de agudização estão gerando forte impacto nos serviços de atenção às urgências e emergências. Compromete-se, dessa forma, os processos de trabalho, a qualidade da assistência e a efetividade das ações em virtude da superlotação e sobrecarga nas portas de entrada. ${ }^{22}$

Logo, o enfermeiro se torna o profissional mais habilitado para realização do atendimento classificatório, visto que esse profissional é detentor de uma visão holística e desenvolve características generalistas que permitem a coordenação da equipe de enfermagem. Assim, perante sua avaliação, consegue elencar quais pacientes têm prioridade e quais podem aguardar em segurança. ${ }^{21}$

Diante dessa CR, realizam-se a entrevista e o acolhimento com o paciente. Busca-se identificar o motivo que o levou ao serviço de saúde, a identificação dos sinais e sintomas e a realização do ECG. Por meio dessas ferramentas, levantam-se as necessidades do paciente com vistas ao estabelecimento e encaminhamentos de condutas conforme relato dos profissionais:

No primeiro momento a gente atende aqui na emergência, aí agora que a gente tem o pessoal da cardio, eles vêm e avaliam aqui, aí se tem alguma 
15 | Zanettini A, Diniz MBC, Stochero G, Silva JC, Marcon C, Cunha VP

identificação que precisa de algo a mais, vai pra UDT e a partir dali eles encaminham no caso se precisar ir para a hemodinâmica, eles que fazem os tramites. (Cava Inferior)

Alteração isquêmica aguda, hemodinâmica, cateterismo de urgência a fim de fazer uma angioplastia primaria, caso seja diagnosticado um infarto agudo do miocárdio, essa é a primeira alteração. Digamos que o paciente fez eletro, chego sem dor, com dor, mas uma dor atípica na sala de emergência, e aí fez o eletro e tem alteração a primeira conduta é encaminhar para a unidade de dor torácica e chamar o cardiologista, o residente da cardiologia pelo menos. (ACD)

O enfermeiro que atua nas portas de entrada hospitalares precisa conhecer suas possibilidades e disponibilidades dentro de cada unidade para poder ter a capacidade de organizar fluxos e direcionar o atendimento ao serviço especializado, como no caso em questão para a UDT. Permite-se, assim, uma ordem cronológica e viável tanto dos usuários quanto para o restante das equipes, de forma a incentivar e aprimorar a padronização de condutas e encaminhamentos.

Cabe salientar que, se o IAM estiver com o intervalo do início do evento $\leq 12$ horas, é possível percorrer dois caminhos conforme a disponibilidade da instituição. Como primeira abordagem surge a angioplastia primária, procedimento em que são avaliadas as coronárias com o intuito de obtenção de fluxo efetivo na coronária afetada, tendo menor índice de complicações tardias, redução de mortalidade em condições específicas, como no choque cardiogênico, e menor período de permanência hospitalar. ${ }^{7}$

A segunda abordagem se refere à aplicação do fibrinolítico. Isso ocorre quando não há hemodinâmica no hospital. No entanto, para sua administração, há necessidade de atentar para as contraindicações, tais como caso o paciente tenha apresentado AVC isquêmico $<3$ meses ou tenha suspeita de dissecção aguda da aorta, sangramento interno ativo, gestação, entre outros, não deve ser aplicado. Caso não se encaixe nas contraindicações e for aplicado o fibrinolítico, 
Dor torácica aguda: enfermeiro desafiando uma patologia tempo dependente nas... 16

devem ser observados os tempos de porta-balão < 90 minutos visando à segurança e melhor efetividade das terapias escolhidas. ${ }^{7}$

O protocolo de dor torácica conduz o atendimento de tal forma que prediz condutas, normas e rotinas para que todos os profissionais o utilizem como meio de informação e norteamento de ações, facilitando, dessa forma, o entendimento do que deve ser avaliado. $\mathrm{Na}$ instituição onde esta pesquisa foi conduzida, a abordagem de escolha majoritária é a intervenção hemodinâmica.

Quando questionados sobre as dificuldades referentes à CR nas portas de entrada, os profissionais identificaram apenas a ideia central de dificuldades estruturais e organizacionais:

Precisaria uma reestruturação organizacional não só equipamentos, equipamentos se tem, mas a questão de enfermagem e a questão médica, necessitaria passar por uma repaginada que se melhorasse algumas coisas, a tecnologia ajudaria muito, mas se tem tecnologia e não tem profissional que domine talvez ela, ou profissionais que intenda algumas necessidades que precisa. (V. Pulmonar)

Eu acho que uma das principais causas é a falta de enfermeiros nas portas de entrada, enfermeiro só para classificação de risco na emergência [...]. O primeiro contato no hospital é o enfermeiro, não adianta, se não tem o enfermeiro disponível só para isso, eu acho que é o nosso maior entrave. (ACD)

Observa-se, a partir dos achados com as dificuldades encontradas no setor de CR, que o profissional enfermeiro mesmo sendo habilitado para o trabalho encontra adversidades no cenário atual. Dentre estas, o quadro de profissionais reduzidos, possível falta de recursos materiais, o alto fluxo de pacientes e falhas na gestão do serviço. ${ }^{23}$

Quanto ao subdimensionamento de pessoal, principalmente no que diz respeito à inexistência de um enfermeiro exclusivo para realização da CR nas portas de entrada, entende- 
se que esse fato é gerador de sobrecarga e estresse. Assim sendo, isso é capaz de refletir diretamente em uma possível avaliação inadequada, resultando em uma CR não fidedigna.

Como limitação, considera-se o fato de a pesquisa ter sido realizada em um único centro, o que pode dificultar a generalização dos resultados para outras populações e regiões. No entanto, os resultados aqui obtidos vislumbram a necessidade da continuidade de pesquisas que versem sobre a temática, uma vez que existem poucos estudos de enfermagem sobre a percepção da atuação do enfermeiro nas portas de entrada, principalmente quando se referem a situações agudas em emergência.

\section{Considerações finais}

A percepção dos enfermeiros diante do paciente com dor torácica passa pelo entendimento de que, na CR nas portas de entrada, as habilidades do enfermeiro estão sistematicamente sendo confrontadas com uma realidade assistencial baseada em protocolos atuais complexos que demandam conhecimento e dimensionamento adequado de pessoal. Sob a ótica do enfermeiro, isso pressupõe que esse profissional reconheça de imediato os sinais e sintomas de um IAM e utilize um fluxograma de atendimento preestabelecido, substituindo a triagem excludente por um modelo de classificação eficaz e de qualidade. Assim, objetiva-se obter uma alta precisão diagnóstica em uma patologia tempo dependente.

Além disso, fica claro para o enfermeiro que o acúmulo da responsabilidade da demanda da gestão com a tarefa assistencial é deletério. Ele ainda desenvolve ações que envolvem a educação em serviço e pesquisas. Com base nos achados desta pesquisa, percebe-se que a priorização das ações de CR das portas de entrada pode estar sendo relegada a um segundo plano, o que foi percebido como verdade pelos enfermeiros.

Os resultados contribuem para a prática assistencial, pois servem de alerta para gestores oportunizarem espaços para discutir e repensar a organização de trabalho na emergência. Isso 
Dor torácica aguda: enfermeiro desafiando uma patologia tempo dependente nas... 18

foi evidenciado na literatura e descrito no presente estudo, perante as falas dos participantes. Algumas delas foram divergentes.

Dessa forma, este estudo contribuirá para a enfermagem ao propor uma reflexão sobre a situação vivida nesse contexto, conhecendo a realidade brasileira quanto à mortalidade pelo IAM e as dificuldades enfrentadas nas portas de entrada hospitalares. Contudo, a presente investigação não apenas revelou alguns nós críticos do processo, mas também vislumbrou a necessidade do cumprimento dos processos de educação em saúde com foco na segurança do paciente, como também sustentação do dimensionamento adequado de profissionais.

\section{Referências}

1. Vieira AC, Bertoncello KCG, Girondi JBR, Nascimento ERP, Hammerschmidt KSA, Zeferinho MT. Perception of emergency nurses in using a chest pain assessment protocol. Texto \& Contexto Enferm. 2016;25(1):1-7. doi: http://dx.doi.org/10.1590/0104-07072016001830014

2. Santos F, Freire PB, Ribeiro JA. Abordagem da dor torácica pelo enfermeiro em uma unidade de pronto atendimento na visão do paciente. Enferm Rev [Internet]. 2016 [acesso em 2018 set 10];19(2):199211. Disponível em: http://periodicos.pucminas.br/index.php/enfermagemrevista/article/view/13149

3. Reggi S, Stefanini E. Diagnóstico das síndromes coronarianas agudas e modelo sistematizado de atendimento em unidades de dor torácica. Rev Soc Cardiol Estado de São Paulo [Internet]. 2016 [acesso em 2018 out 18];26(2):78-85. Disponível em: http://bases.bireme.br/cgibin/wxislind.exe/iah/online/?IsisScript=iah/iah.xis\&src=google\&base=LILACS\&lang=p\&nextAction=lnk\& exprSearch=796510\&indexSearch=ID

4. Teixeira AFJ, Franco A, Castanharo J, Oliveira KCS. Atuação da equipe de enfermagem no atendimento de emergência ao paciente com infarto agudo do miocárdio. Rev Fafibe On Line [Internet]. 2015 [acesso em 2018 set 18];8(1):300-9. Disponível em: http://unifafibe.com.br/revistasonline/arquivos/revistafafibeonline/sumario/36/30102015185545.pdf

5. Servinc S, Akyol AD. Cardiac risk factors and quality of life in patients with coronary artery disease. J Clin Nurs. 2010;19(9-10):1315-25. doi: https://doi.org/10.1111/j.1365-2702.2010.03220.x

6. Medeiros TLF, Andrade PCNS, Davim RMB, Santos NMG. Mortality by an acute myocardial infarction. Rev Enferm UFPE On Line. 2018;12(2):565-72. doi: https://doi.org/10.5205/1981-8963v12i2a230729p565-572-2018

7. Piegas LS, Timerman A, Feitosa GS, Nicolau JC, Mattos LAP, Andrade MD, et al. V Diretriz da 
Sociedade Brasileira de Cardiologia sobre tratamento do infarto agudo do miocárdio com supradesnível do segmento ST. Arq Bras Cardiol [Internet]. 2015 [cited 2020 abr 01];105(2):1-121. Available from: http://publicacoes.cardiol.br/2014/diretrizes/2015/02_TRATAMENTO\%20DO\%20IAM\%20COM\%20SUPR ADESNIVEL\%20DO\%20SEGMENTO\%20ST.pdf

8. Ministério da Saúde (BR), Secretaria de Assistência à Saúde. Programa Nacional de Humanização da Assistência Hospitalar [Internet]. Brasília (DF): Ministério da Saúde; 2001 [acesso em 2019 jul 15]. Disponível em: http://bvsms.saude.gov.br/bvs/publicacoes/pnhah01.pdf

9. Secretaria de Saúde do Distrito Federal (BR). Manual de Acolhimento e Classificação de Risco [Internet]. Brasília (DF): Ministério da Saúde; 2017 [acesso em 2019 jul 15]. Disponível em: http://www.saude.df.gov.br/wp-conteudo/uploads/2017/10/MANUAL-DE-ACOLHIMENTO-ECLASSIFICA\%C3\%87\%C3\%83O-DE-RISCO-DA-REDE-SES-Web.pdf

10. Yin RK. Pesquisa qualitativa do início ao fim. Porto Alegre: Penso; 2016.

11. Tong A, Sainsbury P, Craig J. Consolidated criteria for reporting qualitative research (COREQ): a 32item checklist for interviews and focus groups. Int J Qual Health Care. 2007;19(6):349-57. doi: https://doi.org/10.1093/intqhc/mzm042

12. Bardin L. Análise de Conteúdo. Edições 70. Lisboa (Portugal): LDA; 2011.

13. CONSELHO NACIONAL DE SAÚDE. Resolução no 466, de 12 de dezembro de 2012. Diretrizes e normas regulamentadoras de pesquisas envolvendo seres humanos. Brasília, DF, 2013. Disponível em: http://conselho.saude.gov.br/resolucoes/2012/Reso466.pdf. Acesso em: 26 mar. 2018.

14. Audi CA, Santiago SM, Andrade MD, Francisco PM. Risk factors for cardiovascular disease in prison institution staff: a cross-sectional study. Epidemiol Serv Saúde [Internet]. 2016 [cited 2018 Aug 03];25(2):301-10. Available from: https://www.ncbi.nlm.nih.gov/pubmed/27869948

15. Medeiros TLF de, Andrade PCNS de, Davim RMB, dos Santos NMG. Mortality by an acute myocardial infarction. Rev Enferm UFPE On Line. 2018;12(2):565-72. doi: https://doi.org/10.5205/19818963-v12i2a230729p565-572-2018

16. Piegas LS, Feitosa G, Mattos LA, Nicolau JC, Rossi Neto JM, Timerman A, et al. Sociedade Brasileira de Cardiologia. IV Diretriz da Sociedade Brasileira de Cardiologia sobre tratamento do infarto agudo do miocárdio com supradesnível do segmento ST. Arq Bras Cardiol [Internet]. 2009 [acesso em 2018 set 05];93(6 Supl 2):e179-e264.Disponível em: http://publicacoes.cardiol.br/consenso/2009/diretriz_iam_9306supl2.pdf

17. Santos F, Freire PB, Ribeiro JA. Nurse's approach to chest pain in an emergency care unit in the patient's vision. Enferm Rev [Internet]. 2017 fev [acesso em 2020 mar 31];20(1):13-22. Disponível em: http://periodicos.pucminas.br/index.php/enfermagemrevista/article/view/15411 
18. Gouvêa VET, Reis MAM, Gouvêa GM, Lima HN, Abuabara A. Evaluation of the Manchester Triage System in the Acute Coronary Syndrome. Int J Cardiovasc Sci [Internet]. 2015 [acesso em 2018 set 13];28(2):107-13. Disponível em: http://www.onlineijcs.org/sumario/28/pdf/v28n2a05.pdf

19. Viana RR, Souza MRSM. Marcadores bioquímicos no infarto agudo do miocárdio. Rev Eletrônica Biociências Biotecnologia Saúde [Internet]. 2017 [acesso em 2019 jul 18];9(18):1-8. Disponível em: https://interin.utp.br/index.php/GR1/article/view/1480

20. Reggi S, Carvalho AC. The importance of telemedicine and of pre-hospital thrombolysis. Rev Soc Cardiol Estado de São Paulo [Internet]. 2016 [acesso em 2018 set 20];26(2):86-92. Disponível em: http://socesp.org.br/revista/assets/upload/revista/17635641841534341390pdfL60-REVISTA-SOCESP-V26N2-12-07-16.pdf

21. Camara RF, Paulino TS, Pereira FCC, Nelson ICASR, Rocha KM, Inácio Neto L. O papel do enfermeiro no processo de classificação de risco na urgência: uma revisão. Rev Humano Ser [Internet]. 2015 [acesso 2020 mar 31];1(1):99-114. Disponível em: http://periodicos.unifacex.com.br/humanoser/article/view/628/146

22. O’Dwyer G, Konder MT, Reciputti LP, Macedo C, Lopes MGM. O processo de implantação do Serviço de Atendimento Móvel de Urgência no Brasil: estratégias de ação e dimensões estruturais. Cad Saúde Pública. 2017;33(7):e00043716. doi: https://doi.org/10.1590/0102-311x00043716

23. Abreu JG, Souza KA, Assis EV, Sousa MNA, Silva EN. Dificuldades encontradas pelos profissionais de saúde à implementação da classificação de risco no setor de urgência e emergência. RIS [Internet]. 2016 mar [acesso em 2018 set 17];1(3):209-22. Disponível em: http://www.interdisciplinaremsaude.com.br/Volume_9/Trabalho_12.pdf

\section{Autor correspondente}

Angélica Zanettini

E-mail: angeliica.zanettini@gmail.com

Endereço: Av. São Pedro, Passo dos Fortes- Chapecó.

CEP: 89805 - 120

\section{Contribuições de Autoria}

\section{1 - Angélica Zanettini}

Concepção e planejamento do projeto de pesquisa, análise e interpretação dos dados, redação.

\section{2 - Marisa Basegio Carretta Diniz}

Planejamento do projeto de pesquisa, redação e revisão crítica. 
21 | Zanettini A, Diniz MBC, Stochero G, Silva JC, Marcon C, Cunha VP

\section{3 - Gabriela Stochero}

Redação e revisão crítica.

\section{4 - Juliane Christofari da Silva}

Redação e revisão crítica.

\section{5 - Camila Marcon}

Redação e revisão crítica.

6 - Viviane Pecini da Cunha

Revisão crítica.

\section{Como citar este artigo}

Zanettini A, Diniz MBC, Stochero G, Silva JC, Marcon C, Cunha VP. Dor torácica aguda: enfermeiro desafiando uma patologia tempo dependente nas portas de entrada hospitalares. Rev. Enferm. UFSM. 2020 [Acesso em: Anos Mês Dia]; vol.10 e42: P-21. DOI:https://doi.org/10.5902/2179769236945 